\title{
TESTING THE SENSITIVITY OF SOME TOMATOES VARIETIES TO INFECTION WITH THE PATHOGEN ALTERNARIA ALTERNATA KEISSLER , AND THE EFFECT OF SODIUM BICARBONATE IN FUNGAL GROWTH IN VITRO Saad Aldean, A. ${ }^{1}$; W. Nafaa $^{2}$ and Taiser Abu Alfadhel ${ }^{3}$ 1-Qunietra countryside
ahmadsaedaldean@gmail.com center for (S.A.R).
}

2- Plant Protection Dept, Fac. of Agric. , Damascus University.

3- Directorate of Plant Protection, Ministry of Agric. and Agric. Reform

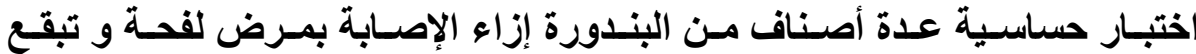

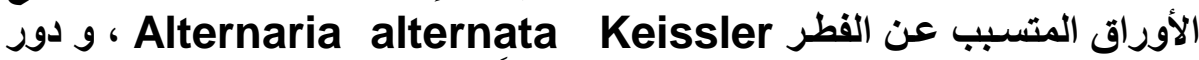

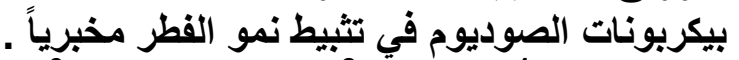

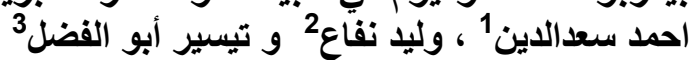

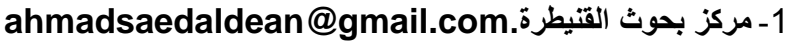

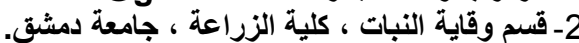
3- مديرية وقاية المزروعات ـ وزارة الزراعة و الإصلاح الزراعي ـ

الملخص

نفذت هذه الدراسة في المخابر التابعة لإدارة بحوث وقاية النبات في الهيئة العامة للبحوث العزبة العلمية

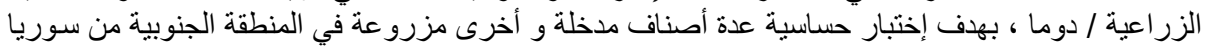

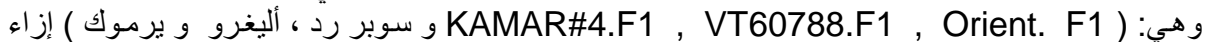

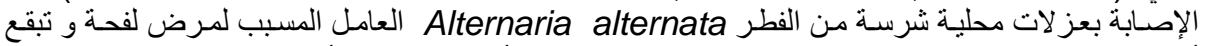

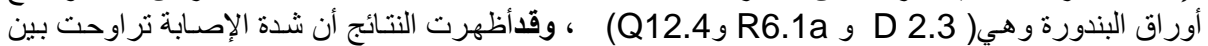

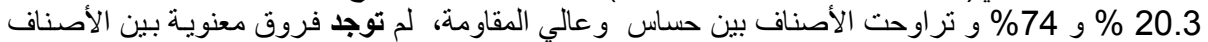

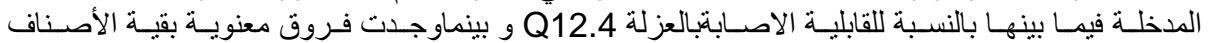

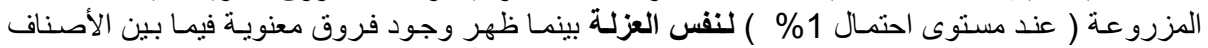

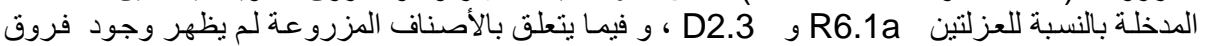

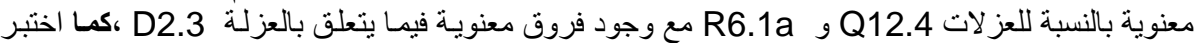

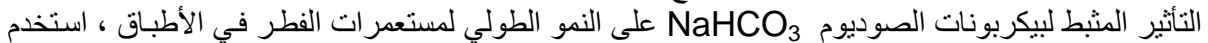

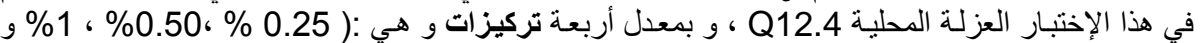

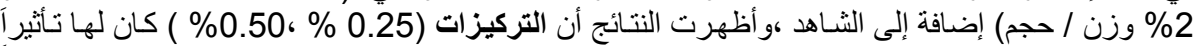

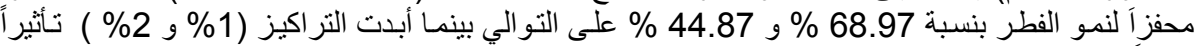

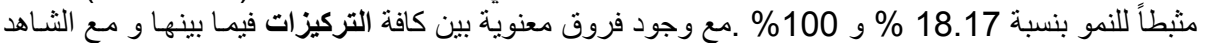

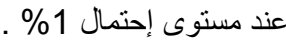
الكلمات المفتاحية: البندورة - لفحة و تبقع الأوراق - حساسية الأصناف ـ بيكربونات الصوديوم . المقدمة

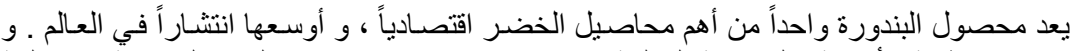

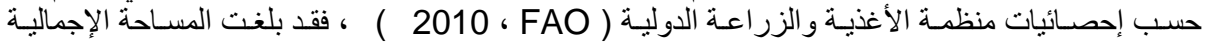

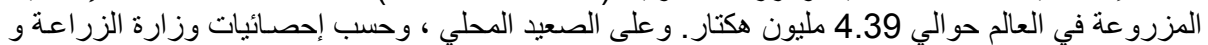

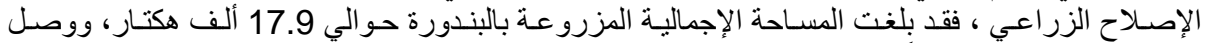

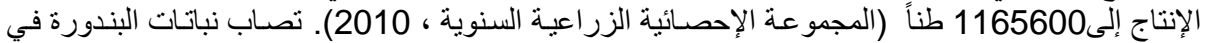

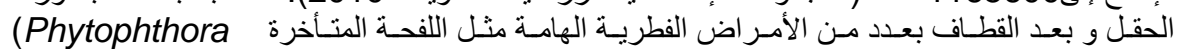

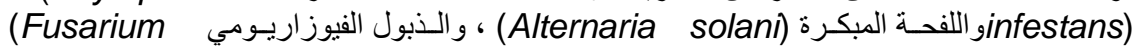




\section{Saad Aldean, A. et al.}

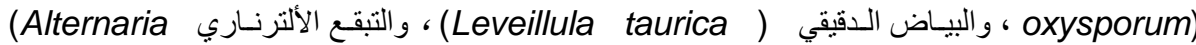
( Rhizoctonia solani (alternata)

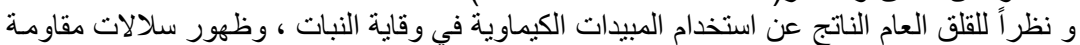

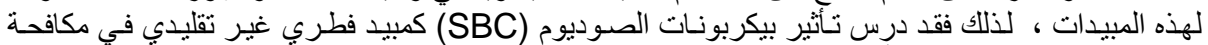

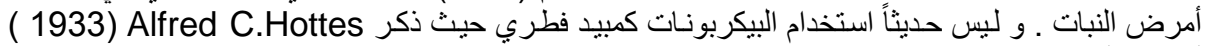

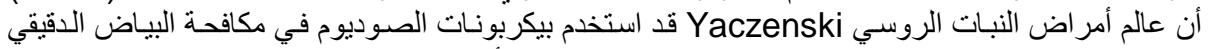

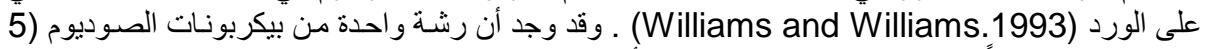

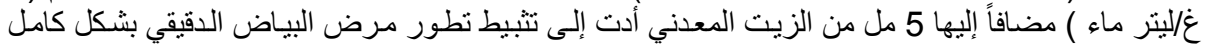

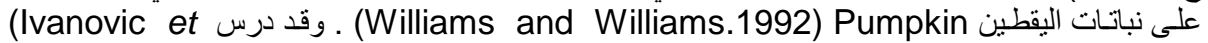

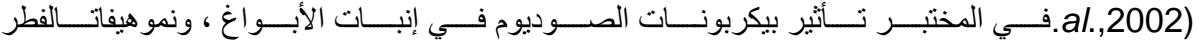
Alternaria.solani

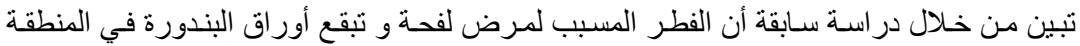

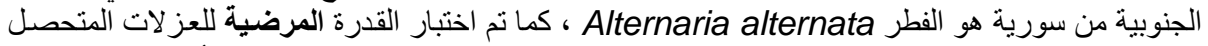

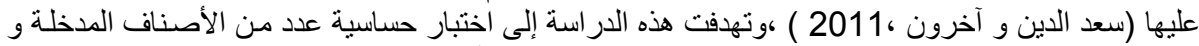

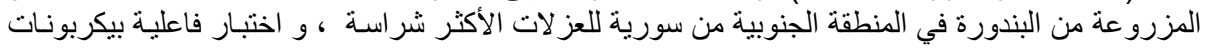

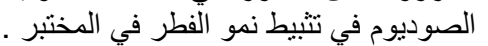
2-2 - مواد البحث و طر ائقه : 1-2 - اختبار حساسية أصناف البندورة للإصابة :

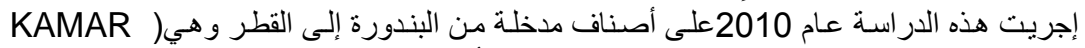

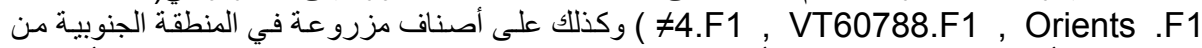

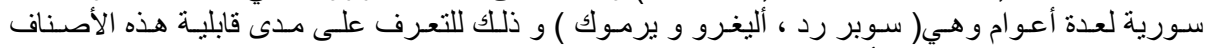

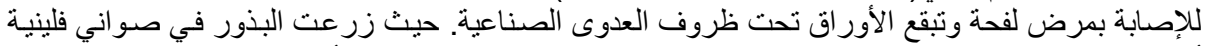

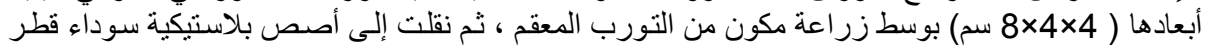

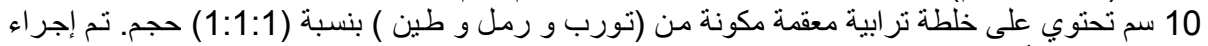

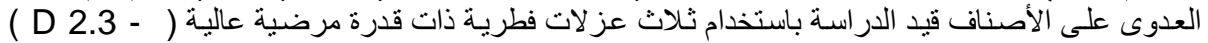

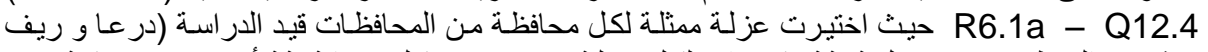

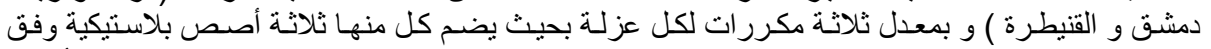

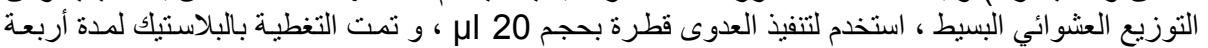

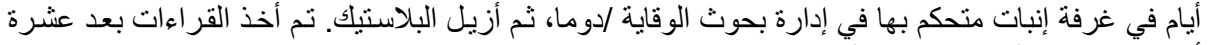

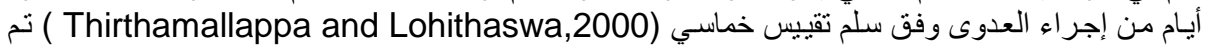

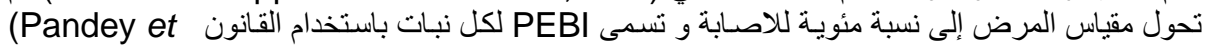
: al,2003)

\section{PEBI $=\frac{\text { sum off all ratings }}{\text { sum }}$ no. of leaves $\mathbf{s} \square$ mpled $*$ maximum disease scale * 100}

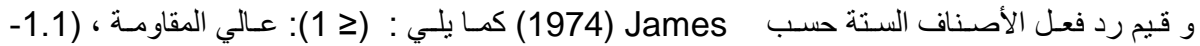
2):مقاوم ، (2.1-3): منوسط المقاومة،(3.1 - 4):قابل للإصابة ، (4.1-5 ):حساس . .

جدول 1: سلم تقييس شدة إصـابة أصناف البندورة بمرض تبقع الأوراق الألترنـاري المتسبب عن الفطر . Alternaria alternata

\begin{tabular}{|c|c|c|}
\hline \multicolumn{2}{|r|}{ طبيعة الإصابة } & درجة سلم \\
\hline قطر البقعة (مم) & النسبة المئوية للمساحة المنكرزة (أو المصابة) من الورقة & التقييس \\
\hline لا يوجد بقع & $\% 0$ & 1 \\
\hline > & \% من 10 > & 2 \\
\hline $5-2$ & $\% 25-10$ & 3 \\
\hline
\end{tabular}




\begin{tabular}{|c|c|c|}
\hline $7.5-5$ & $\% 49-26$ & 4 \\
\hline $7.5<$ & $\% 100-50$ & 5 \\
\hline
\end{tabular}

2-2-2

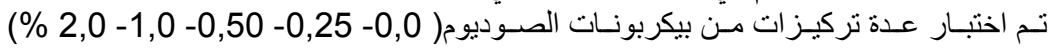

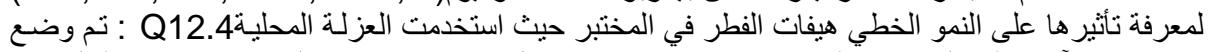

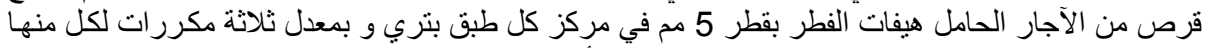

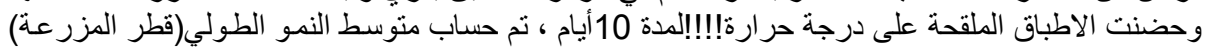
للفطر بعد 10 أيام ( 2007, Abd-El-Kareem ) . تم حساب النسبة المئوية للتبيط النمو القطري للفطر وفق معادلة (Hinderson and Tiltton, 1955)

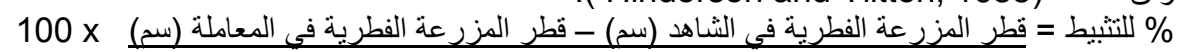
قطر المزرعة الفطرية في الثناهد (سم) الفزرعية

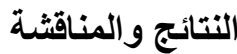

1-3-3 اختبار حساسية أصناف البندورة للإصابة بالمرض:

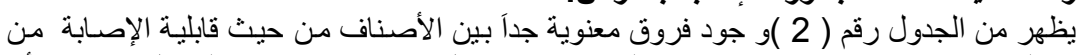

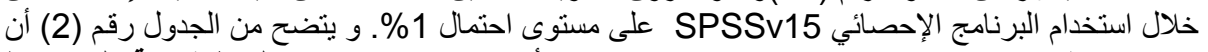

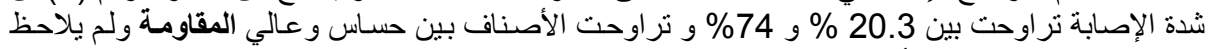

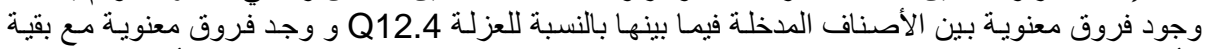

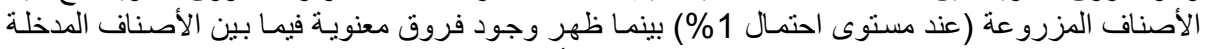

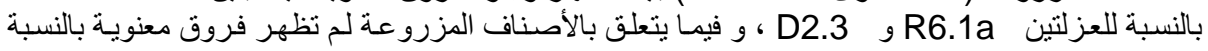

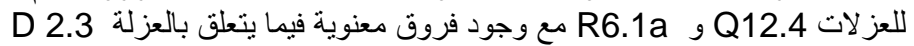

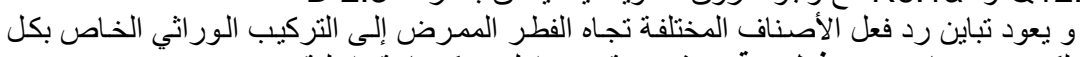

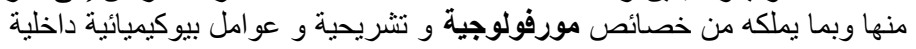

(Latin et al,1994; Latin and Evans, 1996; Egel, 1999) 2-3- فاعلية بيكربونات الصوديوم في تثبيط نمو الفطر في المختبر:

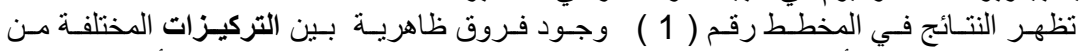

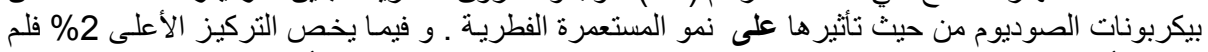

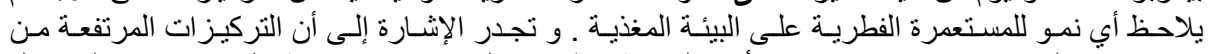

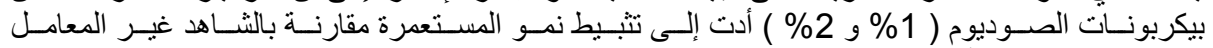

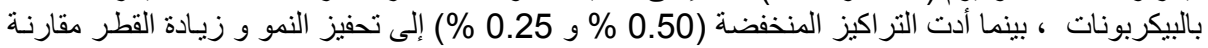

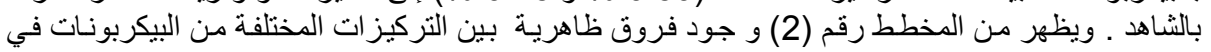

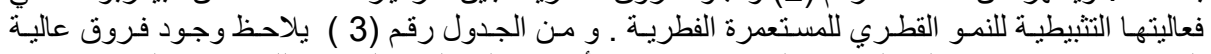

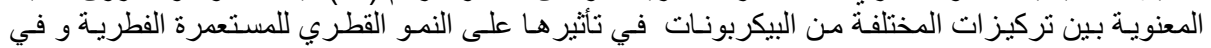

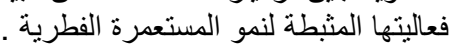

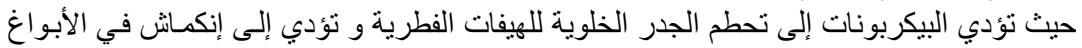

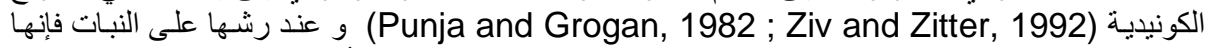

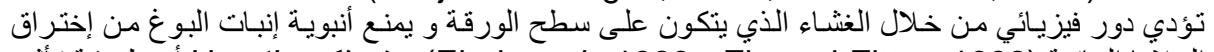

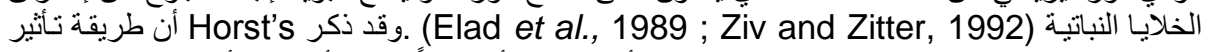

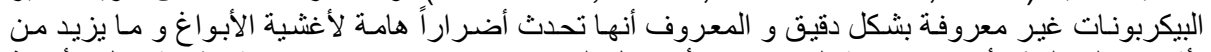

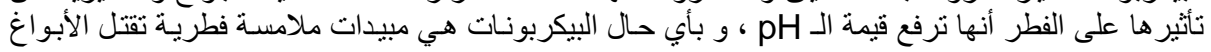

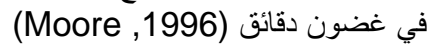


Saad Aldean, A. et al.

(1) المخطط

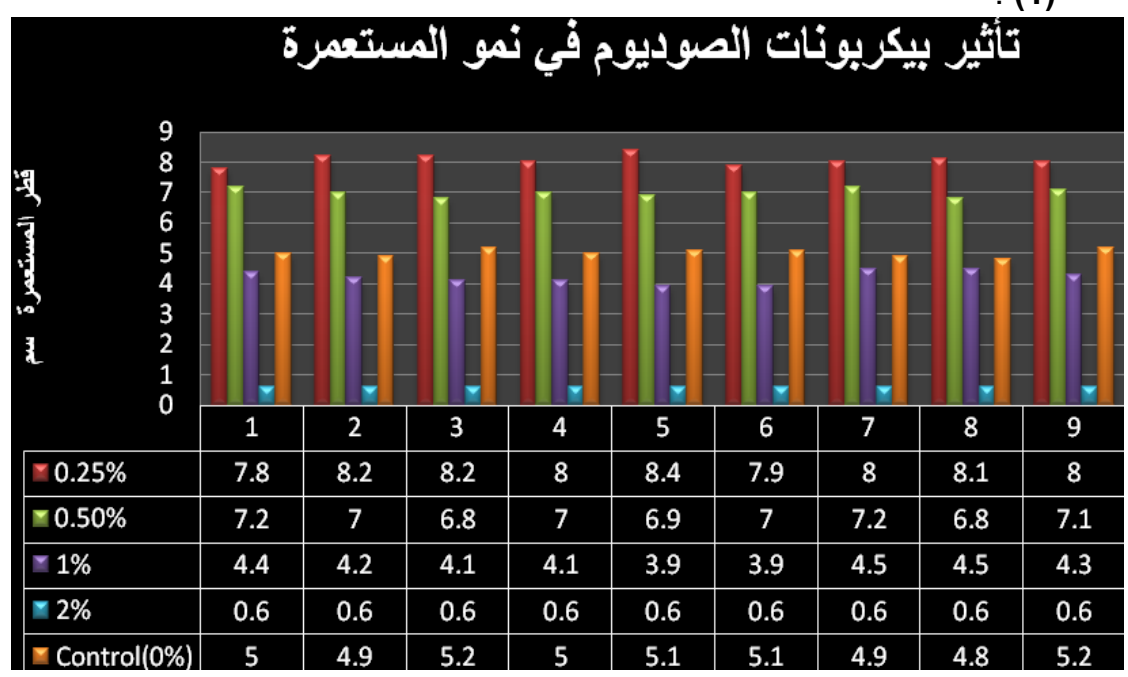

المخطط ( 2 ):

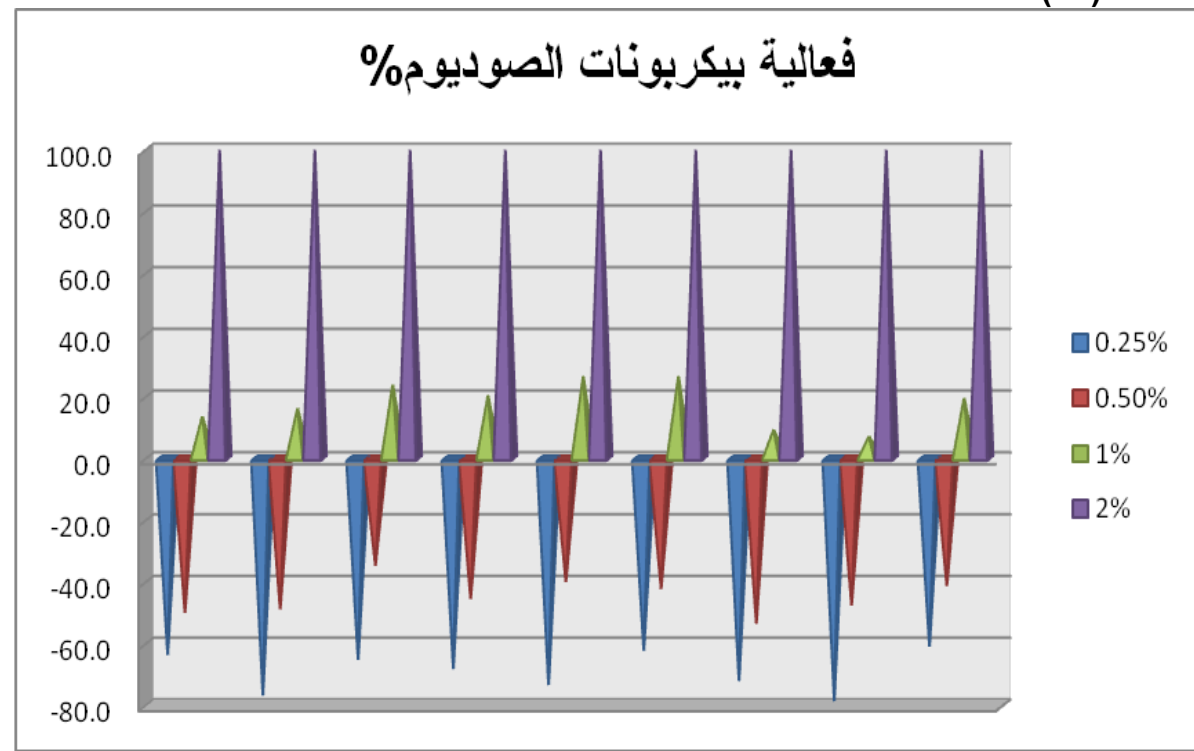

جدول 2 : النسبة المئوية لثدة إصابة أصناف البندورة المختبرة بالعزلات المحلية :

\begin{tabular}{|c|c|c|c|c|c|c|c|c|c|}
\hline \multicolumn{3}{|c|}{ العزلة D2.3 } & \multicolumn{3}{|c|}{ العزلة R 6.1a } & \multicolumn{3}{|c|}{ العزلة Q12.4 } & \multirow[b]{2}{*}{ الصنف } \\
\hline الصنفية & الإصابة & شدة الإصابة & حساسية الصنف| & لاصرجة & ثدة الإصابة \%\% & |حسنفية & الإصابة & شدة الإصابة \% | & \\
\hline عالي المقاومة & 0.6 & $25.33 \mathrm{a}$ & متوسط مقاومة | & 2.5 & $54.67 a$ & | المقاومَّة & 0.1 & $20.3 \mathrm{a}$ & KAMAR\#4.F1 \\
\hline عالي المقاومة & 0.7 & $26.37 a$ & عالي المقاومة & 0.4 & $21 . b$ & مقاوم & 1.4 & $28 a$ & VT60788.f1 \\
\hline
\end{tabular}




\begin{tabular}{|c|c|c|c|c|c|c|c|c|c|}
\hline متو سط مقاو مة & 2.3 & $49 b$ & عالى المقاومة & 0.4 & $23.23 \mathrm{~cd}$ & |المقاومة & 0.3 & $22 a$ & Orient.f1 \\
\hline حساس & 4.2 & 72.7ce & قابل للإِصابة & 4.0 & $70.79 d$ & حساس & 4.3 & 63.03de & سوبر رد \\
\hline قابل للإصدابة & 3.3 & $56.6 \mathrm{~d}$ & قابل للإصـابة & 4.0 & $68 \mathrm{ed}$ & مقاومة & 2.8 & $60.17 \mathrm{ed}$ & | أليغرو \\
\hline قابل للإصابة & 3.1 & $68.3 \mathrm{e}$ & قابل للإصـابة & 3.7 & $70.03 \mathrm{fd}$ & للإصابة & 3.3 & $61.67 \mathrm{fde}$ & يرموك \\
\hline
\end{tabular}

الجدول 3 : تأثثير التراكيز المختلفة مـن بيكربونـات الصـوديوم فى النمـو الخطى للمستعمرة الفطريسه وفى فاعليتها المثبطة

\begin{tabular}{|c|c|c|}
\hline التثبيط \% & النمو القطري سم & \% NaHCO3 تركيز \\
\hline$-68.97^{a}$ & $7.47^{\mathrm{a}}$ & 0.25 \\
\hline$-44.87^{b}$ & $6.4^{\mathrm{b}}$ & 0.50 \\
\hline $18.17^{\mathrm{C}}$ & $3.61^{c}$ & 1 \\
\hline $100^{d}$ & $0^{\mathrm{d}}$ & 2 \\
\hline & $4.42^{\dagger}$ & Control \\
\hline
\end{tabular}

- ـ القيم المتبوعة بأحرف متثابهة ضمن العمود الواحد لا يوجد بينها فروق معنوية عند مستوى احتمال 1\%.

و قد وجد الاخيل (2009) أن إضافة الأحماض(ساليسيليك SA و بنزويك BA و الأكساليك OA

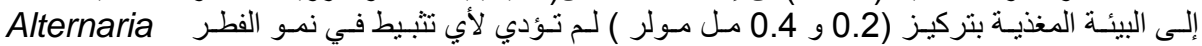
cucumerina

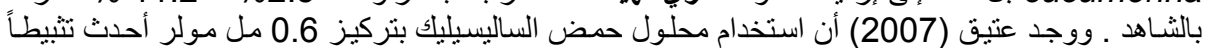
بنسبة 23.8 \% في نمو الفطر Alternaria solani و 15.5 \% في نمو الفطر A. alternata فئرل

$$
\text { المراجع }
$$

المجموعة الإحصائية الزراعية السنوية 2010 ـ المكتب المركزي للإحصـاء ـالجمهوريـة العربية السورية

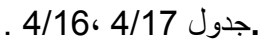

عتيق،عمر.أحمد الأحمد،محمد أبو شعر ،محمد موفق يبرق. 2007.مستح حقلي لأمر اض البندورة /الطماطم

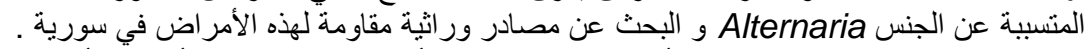

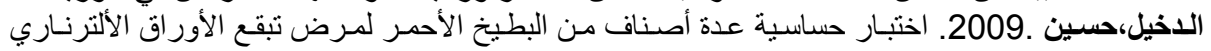

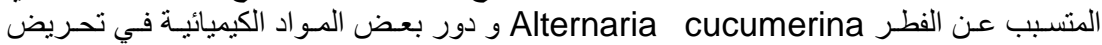

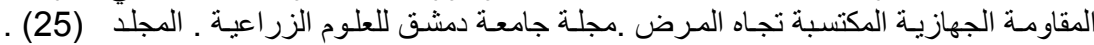

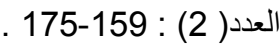

F. Abd-El-Kareem .2007. Potassium or Sodium Bicarbonate in Combination with Nerol forControlling Early Blight Disease of Potato Plants under Laboratory,Greenhouse and Field Conditions. Plant Pathol. Dept., National Res. Centre, Giza, Egypt. Egypt. J. Phytopathol., Vol. 35, No. 1, pp. 73-86 (2007).

Egel, D. S. (1999). Severity of Alternaria leaf blight on muskmelon varieties ,Biol. Cult. Tests 14:157.

Elad, Y.; Zive, O.; Ayash, N. and Katan, J. 1989. The effect of film forming polymers on powdery mildew of cucumber. Phytoparasitica, 17: 179288.

FAO. 2010., FAOSTAT statical data base.

Henderson, C.F. and E. W. Tilton, 1955. Tests with acaricides against the brow wheat mite, J.Econ. Entomol. 48:157-161. 


\section{Saad Aldean, A. et al.}

Ivanovic, M.; Mijatovic, M.; Antonijevic, D.;2002. Effect of sodium bicarbonate on Alternaria solani in tomato .ACTA HORTICULTURAE, no.579:535539, 2002.

James, W. C. (1974). Assessment of plant diseases and losses Annula Review of Phytopathology , 12, 27-48.

Latin, R. X. and Evans, K. J. (1996). Development and delivery of a forecaster for Alternaria leaf blight of muskmelon. [Abstr.] Phytopathology 86:S106.

Latin, R., Rane, K. K. and Evans, K. J. (1994). Effect of Alternaria leaf blight on soluble solid content of muskmelon. Plant Dis.78:979-982.

Moore, S. R. 1996. Bicarbonates offer effective disease control. Grower Talks.February. p. 72.

Pandey KK, Pandey PK, Kallo G, Banerjee MK. 2003. Resistance toearly blight of tomato with respect to various parameters of disease epidemics. J Gen Plant Pathol 69:364-371.

Punja, Z. and Grogan, R.G. 1982. Effects of inorganic salts carbonatebicarbonate anions, ammonia and the modifying influence of $\mathrm{pH}$ on sclerotia germination of Sclerotium rolfsii. Phytopathology, 72: 635639.

Thirthamallappa and H. C. Lohithaswa. (2000). Genetics of resistance to early bloght [Alternaria solani Sorauer] in tomato [ Lycoperrsicon esculentum L .] Euphytica 113: 187-193.

Warnock, S.J. 1991 .Natural habitats of lycopersicum species . Hort. Science ,26, 466-471.

Williams, G. and P. Williams. 1992. More on baking soda/horticultural oil vs.fungal disease. Hortldeas. June. p. 69.

Williams, G. and P. Williams. 1993. Baking soda vs. powdery mildew: Not a neidea! Hortldeas. June. p. 62.

Ziv, O. and T. A. Zitter. 1992. Effects of bicarbonates and film-forming polymerson cucurbit foliar diseases. Plant Disease. Vol. 26, No. 5. p. 513-517. 


\title{
TESTING THE SENSITIVITY OF SOME TOMATOES VARIETIES TO INFECTION WITH THE PATHOGEN ALTERNARIA ALTERNATA KEISSLER , AND THE EFFECT OF SODIUM BICARBONATE IN FUNGAL GROWTH IN VITRO Saad Aldean, A. ${ }^{1}$; W. Nafaa ${ }^{2}$ and Taiser Abu Alfadhel ${ }^{3}$ 1-Qunietra countryside
ahmadsaedaldean@gmail.com center for \\ (S.A.R).
}

2- Plant Protection Dept, Fac. of Agric. , Damascus University.

3- Directorate of Plant Protection, Ministry of Agric. and Agric. Reform

\begin{abstract}
ABSRTACT
This study conducted in the laboratories of General Commission for Agriculture Research( GCSAR) /Doma ,in order to determinate the sensitivity of some tomato varieties (KAMAR\#4.F1, VT60788.F1, Orient. F1, Super red , Alegro and Yarmok) -inputted and cultivated - in southern region of Syria to infection of local isolates of the pathogen Alternaria alteranta ( D 2.3- R6.1a - Q12.4) the major agent of blight and leaf spot of tomato. The results shows that the severity of infection ranged between $20.3 \%$ to $74 \%$. The cultivars ranged from sensitive to high resistance, there is no significant differences between inputted varieties with each other for the isolate Q12.4(with probability level 1\%), and there is a significant differences with the rest of varieties, on the other hand, the results showed that the significant differences existed between the inputted varieties for the isolates $R 6.1 \mathrm{a}$ and D2.3 , and with the cultivated varieties did not show a significant differences between the isolates Q12.4 and R6.1a , but the significant differences existed for the isolate Q12.4 . the inhibitory effect of sodium bicarbonate $\left(\mathrm{NaHCO}_{3}\right)$ in fungal linear growth tested for the fungus colonies in dishes by using a local isolate Q12.4 with four concentrations of $\mathrm{NaHCO}_{3}(0.25 \%, 0.50 \%, 1$ $\%$ and $2 \%$ W/V in addition to the control. The results showed that concentrations $(0.25 \%, 0.50 \%)$ had a catalytic effect for fungal growth by $68.97 \%$ and $44.87 \%$ respectively , while ,indicate concentrations ( $1 \%, 2 \%$ ) had an inhibitory effect to the growth by $18.17 \%$ and $100 \%$ respectively , with the presence of significant differences between themselves ,and with the control at the level of probability $1 \%$.

keywords : Tomato, Spot and leaf blight, Sensitivity of Varieties ,Sodium bicarbonate.
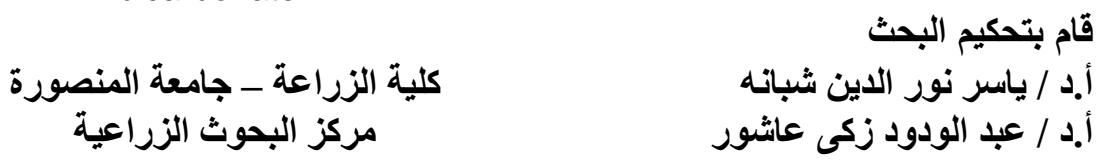